\title{
TOPOLOGICAL ALGEBRAS AND MACKEY TOPOLOGIES
}

\author{
ALLAN C. COCHRAN
}

Abstract. Let $E$ be a locally $m$-convex algebra with dual space $E^{\prime}$. In a recent paper S. Warner asked if the finest locally $m$-convex topology on $E$ compatible with $E^{\prime}$ was the mackey topology. It is shown that this is not the case. A similar result is given for this question in the $A$-convex algebra case. For any $A$-convex algebra, a construction is given of an associated locally $m$ convex algebra. It is shown that this associated locally $m$-convex topology is always the compact-open topology for the space $C_{b}(S)$ with the strict topology.

Seth Warner [9] extended the idea of bornological linear space to the case of locally $m$-convex algebras. For a given locally $m$-convex algebra $E$ with dual space $E^{\prime}$, he noted the existence of a finest locally $m$-convex topology, $\chi\left(E, E^{\prime}\right)$, compatible with the given duality. In this note we show that $\chi\left(E, E^{\prime}\right)$ does not necessarily coincide with the mackey topology $\tau\left(E, E^{\prime}\right)$. This answers a question presented by Warner [9, p. 215, Question 3]. The class of $A$-convex algebras introduced in [3] and [4] provide a similar situation. There is a finest $A$-convex topology, $\Sigma\left(E, E^{\prime}\right)$, compatible with a given duality and we show that $\Sigma\left(E, E^{\prime}\right)$ is not necessarily a mackey topology.

We give a method to construct the finest locally $m$-convex topology coarser than a given $A$-convex topology. Let $S$ denote a locally compact hausdorff space, $C_{b}(S)$ the space of bounded continuous complexvalued functions on $S, \beta$ the strict topology introduced by Buck [2] and $\kappa$ the compact-open topology. We use the description obtained to show that the finest locally $m$-convex topology coarser than $\beta$ is precisely $\kappa$. Thus, there are no locally $m$-convex topologies between $\beta$ and $\kappa$.

2. Preliminaries. In this section the basic definitions are given and a description of the strict topology is listed for use in $\S 3$. Throughout this note $E$ will denote an algebra over $R$ or $C$ and topology will always mean locally convex linear topology.

(2.1) Definition. A convex balanced absorbing subset $V$ of $E$ is called m-convex if $V \cdot V \subset V$ (i.e. if $V$ is idempotent). A convex bal-

Received by the editors June 27,1970 .

AMS 1968 subject classifications. Primary 4650; Secondary 4601, 4625, 1620.

Key words and phrases. Bornological space, mackey topology, locally $m$-convex algebra, $A$-convex algebra, strict topology, compact-open topology. 
anced absorbing subset $V$ of $E$ is called $A$-convex if, for every $x$ in $E$, $V$ absorbs $x V$ and $V x$.

(2.2) Definition. A locally $m$-convex algebra is an algebra $E$ with a topology which has a neighborhood base at zero of $m$-convex sets.

(2.3) Definition. An $A$-convex algebra is an algebra $E$ with a topology which has a neighborhood base at zero of $A$-convex sets.

For information about locally $m$-convex algebras see [6], [1], [8] and [9]; for $A$-convex algebras see [3] and [4]. An equivalent definition of $A$-convex algebra is the following: an $A$-convex algebra is an algebra $E$ with a topology defined via a family $P$ of seminorms such that for $p$ in $P$ and $x$ in $E$, there are constants $M(p, x)$ and $N(p, x)$ such that

(i) $p(x y) \leqq M(p, x) p(y)$, for all $y$ in $E$;

(ii) $p(y x) \leqq N(p, x) p(y)$, for all $y$ in $E$.

It is clear that the class of $A$-convex algebras includes the class of locally $m$-convex algebras and, in particular, all Banach algebras.

(2.4) ExAmple. Let $S$ denote a locally compact hausdorff space, $C_{b}(S)$ the algebra of all bounded continuous complex-valued functions on $S$ and $C_{0}^{+}(S)$ the set of all nonnegative continuous real-valued functions on $S$ which vanish at infinity. The strict topology, $\beta$, is defined in terms of the family of seminorms $\left\{p_{\phi}: \phi \in C_{0}^{+}(S)\right\}$,

$$
p_{\phi}(f)=\sup \left\{|f(x) \phi(x)|: x \in S, f \in C_{b}(S)\right\} \text {. }
$$

For $S=R,\left(C_{b}(R), \beta\right)$ is a complete $A$-convex algebra with identity which is not locally $m$-convex (see [3]).

Other examples may be constructed using the generalization of Example 2.4 called weighted spaces ([3], [4] and [10]).

3. Main results. Let $E$ denote an $A$-convex algebra with $N$ a neighborhood base at zero consisting of $A$-convex sets. Warner [9] proved that the smallest idempotent set containing a given set $T$ is $\bigcup\left\{T^{n}: n=1,2, \cdots\right\}$. For each $V$ in $N$, let $V^{*}$ denote the balanced convex hull of $U\left\{V^{n}: n=1,2, \cdots\right\}$. Since $V$ is absorbing, $V^{*}$ is $m$ convex. Let $N^{*}=\left\{V^{*}: V \in N\right\}$. Then $N^{*}$ is a neighborhood base at zero for an $m$-convex topology on $E$.

(3.1) Lemma. Let $(E, \Phi)$ be an $A$-convex algebra with $N$ a neighborhood base at zero of $A$-convex sets. Then $N^{*}$ determines a locally $m$ convex topology $\Phi^{*}$ which is the finest locally $m$-convex topology coarser than $\Phi$.

Proof. The proof is an easy consequence of the fact that $V^{*}$ is the smallest $m$-convex set containing $V$. 
A neighborhood base of $A$-convex sets for $\left(C_{b}(S), \beta\right)$ is given by

$$
\left\{V(T(\phi), \theta(\phi)): \phi \in C_{0}^{+}(S)\right\}
$$

where $T(\phi)=\{x \in S: \phi(x) \neq 0\}, \theta(\phi): T(\phi) \rightarrow R^{+}$such that $\theta(\phi)(x)$ $=1 / \phi(x)$ and $V(T(\phi), \theta(\phi))=\left\{f \in C_{b}(S):|f(x)| \leqq \theta(\phi)(x)\right.$, for all $x \in T(\phi)\}$.

(3.2) TheOREM. The associated locally m-convex topology on $C_{b}(S)$ for $\beta$ is $\kappa$.

Proof. Note that $[V(T(\phi), \theta(\phi))]^{*}=V(T, \eta)$ where $T=$ $\{x \in T(\phi): \theta(\phi) \leqq 1\}$ and $\eta=\theta \mid T$. Then $T=\{x \in S: \phi(x) \geqq 1\}$ which is a compact subset of $S$ since $\phi \in C_{0}^{+}(S)$. Thus, $[V(T(\phi), \theta(\phi))]^{*}$ is a $\kappa$-neighborhood of zero for each $\phi \in C_{0}^{+}(S)$. It is well known that $\beta \geqq \kappa$ and $\kappa$ is locally $m$-convex. Hence the associated topology for $\beta$ is $\kappa$.

(3.3) Corollary. For a locally compact Hausdorff space $S$, there are no locally m-convex topologies on $C_{b}(S)$ between $\beta$ and $\kappa$.

The following result gives a solution to Warner's Question 3 of [9]. Let $S_{0}$ denote the space of ordinals less than the first uncountable ordinal $\Omega$ with the order topology. Conway [5] has shown that $\beta$ is not a mackey topology on $C_{b}\left(S_{0}\right)$. Morris and Wulbert [7] have shown that $\kappa$ is not mackey and, in fact, a result of Wang [11] shows that $\beta=\kappa$. Let $B=\left\{f \in C_{b}\left(S_{0}\right):|f(x)| \leqq 1\right.$ for all $\left.x \in S_{0}\right\}$. It is known that $B$ is not a neighborhood of zero for the mackey topology $\tau$. In fact, for $f \in C_{b}\left(S_{0}\right)$, there exists $x_{0} \in S_{0}$ with $f(y)=a_{f}$ for $y \geqq x_{0}$. The linear functional defined by $L(f)=a_{f}$ is not in the $\beta$-dual but is bounded on $B$. For $x \in S_{0}$ let $h_{x}(f)=f(x), f \in C_{b}\left(S_{0}\right)$. Then $h_{x}$ is in the $\beta$-dual. The set $V$ defined by the closed balanced convex hull of $\left\{h_{x+1}-h_{x}: x \in S_{0}\right\}$ is weakly compact but not equicontinuous [5], [7]. Thus, $V^{0}$ is a $\tau$-neighborhood of zero but not a $\kappa$ (or $\beta$ ) neighborhood.

(3.4) Theorem. The space $\left(C_{b}\left(S_{0}\right), \tau\right)$ is not locally $m$-convex, where $\tau$ denotes the mackey topology compatible with $\kappa$.

Proof. The $\tau$-neighborhood $W=V^{0}$ of zero defined above does not contain an $m$-convex $\tau$-neighborhood of zero: Suppose $H$ is $m$-convex, $H \subset W$. Let $f \in H$ and $x \in S_{0}$ with $f(x+1) \neq f(x)$. Then $|f(x)| \leqq 1$ since $f^{n} \in H \subset W, n=1,2, \cdots$, and $|f(x)|>1$ gives a contradiction to $\left|f^{n}(x+1)-f^{n}(x)\right| \leqq 1$. If $H$ is a $\tau$-neighborhood of zero, $H$ absorbs the $\tau$-bounded set $B$. Then if $f(x)=f(x+1)$ and $|f(x)|>1$ we use the convexity of $H$ to obtain a function $g$ with $|g(x)|>1$ and $g(x)$ 
$\neq g(x+1)$. By the first part of the proof this is impossible. Hence $H \subset B$ so $B$ must be a $\tau$-neighborhood of zero. But $B$ is not a $\tau$-neighborhood of zero and hence $W$ does not contain an $m$-convex $\tau$-neighborhood of zero. This completes the proof.

The finite intersection of $A$-convex sets is an $A$-convex set. Thus, the supremum of $A$-convex topologies is $A$-convex. Whenever $(E, \Phi)$ is an $A$-convex algebra with dual $E^{\prime}$ there is a finest $A$-convex topology $\Sigma\left(E, E^{\prime}\right)$ on $E$ which is compatible with the pair $\left(E, E^{\prime}\right)$. We now answer the obvious extension of the problem of Warner to the $A$ convex case.

(3.5) Theorem. The space $\left(C_{b}\left(S_{0}\right), \tau\right)$ is not $A$-convex.

Proof. Suppose $H$ is an $A$-convex $\tau$-neighborhood of zero with $H \subset W=V^{0}$. Let $x \in S_{0}$. Then there exists a constant $K$ such that $|f(x)| \leqq K$ for all $f$ in $H$. Suppose such a $K$ does not exist and let $g \in C_{b}\left(S_{0}\right)$ with $|g(x+1)-g(x)|=1$ and $g(x)>1$. Then $g H \subset M H$. Using the convexity of $H$ and the fact that $H$ absorbs $B$, there exists $\sigma>0$ such that for any $L>0$ there exists $f \in H$ with $|f(x)-f(x+1)|$ $\geqq \sigma$ and $|f(x)|>L$. This gives a contradiction to $g f \in M V^{0}$ for all $f$ in $H$.

Let $A(x)=\inf \{M:|f(x)| \leqq M$ for all $f$ in $H\}$. Then $A(x)$ is finite for each $x$ in $S_{0}$. Suppose $A$ is not bounded above. Then there is a sequence $\left\{x_{n}\right\}$ in $S_{0}$ of distinct elements with $A\left(x_{n}\right) \geqq n, n=1,2, \cdots$. Since $S_{0}$ is sequentially compact there is a convergent subsequence. We denote this subsequence by $\left\{x_{k}\right\}$ and the limit by $x_{0}$. There exists some $f \in H$ and a neighborhood of $x_{0}, N$, with $\left|f(x)-A\left(x_{0}\right)\right| \leqq 1$ for $x$ in $N$ (by continuity of $f$ and definition of $A$ ). Also, there is an integer $K$ such that if $n \geqq K$ then $x_{n} \in N$. But $\left\{A\left(x_{n}\right)\right\}$ is unbounded, so by convexity of $H$ there is a function $g \in H$ such that $\left|g\left(x_{n}\right)-g\left(x_{n+1}\right)\right|$ $>1$ contrary to $g \in V^{0}$. Thus, $A$ is bounded above so that some multiple of $B$ contains $H$. This implies that $B$ is a $\tau$-neighborhood of zero which is a contradiction. Thus $\tau$ is not $A$-convex.

It is interesting to observe that for $S=R$, there are no locally $m$ convex topologies in the mackey spectrum of $\left(C_{b}(R), \beta\right)$. This result follows from Theorem 3.2 and the fact that the weak topology of $\left(C_{b}(R), \beta\right)$ is not locally $m$-convex $[4]$.

We conclude this note with the following two unresolved questions.

(3.6) Question. Let $E$ be an algebra and $E^{\prime}$ a subspace of the dual (algebraic) $E^{*}$. If there are both $A$-convex and locally $m$-convex topologies compatible with $\left(E, E^{\prime}\right)$ then must $\Sigma\left(E, E^{\prime}\right)=\chi\left(E, E^{\prime}\right)$ ?

(3.7) Question. Under what conditions, in terms of $E^{\prime}$, does $\Sigma\left(E^{\prime}, E\right)$ and/or $\chi\left(E, E^{\prime}\right)$ exist? 


\section{REFERENCES}

1. R. F. Arens, A generalization of normed rings, Pacific J. Math. 2 (1952), 455471. MR 14, 482.

2. R. C. Buck, Bounded continuous functions on a locally compact space, Michigan Math. J. 5 (1958), 95-104. MR 21 \#4350.

3. A. C. Cochran, C. R. Williams and R. Keown, On a class of topological algebras, Pacific J. Math. 34 (1970), 17-25.

4. A. C. Cochran, Weak A-convex algebras, Proc. Amer. Math. Soc. 26 (1970), 73-77.

5. J. B. Conway, The strict topology and compactness in the space of measures, Bull. Amer. Math. Soc. 72 (1966), 75-78. MR 32 \#509.

6. E. A. Michael, Locally multiplicatively-convex topological algebras, Mem. Amer. Math. Soc. No. 11 (1952). MR 14, 482.

7. P. D. Morris and D. E. Wulbert, Functional representations of topological algebras, Pacific J. Math. 22 (1967), 323-337.

8. S. Warner, Weak locally multiplicatively-convex algebras, Pacific J. Math. 5 (1955), 1025-1032. MR 17, 876.

9. - Inductive limits of normed algebras, Trans. Amer. Math. Soc. 82 (1956), 190-216. MR 18, 52.

10. W. H. Summers, $A$ representation theorem for biequicontinuous completed tensor products of weighted spaces, Trans. Amer. Math. Soc. 146 (1969), 121-131. MR 40 \#4748.

11. J. Wang, Multipliers of commutative Banach algebras, Pacific J. Math. 11 (1961), 1131-1149.

University of Arkansas, Fayetteville, Arkansas 72701 